\title{
Retail health clinics: What is their role in ObGyn care?
}

\author{
Retail health clinics provide improved access to episodic care, and half of all \\ visits are by women
}

\author{
Nate A. Bronstein, MsED, MPA, MSSP, and William F. Rayburn, MD, MBA
}

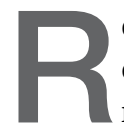
etail Health Clinics (RHCs) are health care facilities located in high-traffic retail outlets with adjacent pharmacies that are intended to provide convenient and affordable care without sacrificing quality. The clinics add an option that complements services to individuals and families who otherwise would need to wait for an appointment with a traditional primary care physician or provider. ${ }^{1}$ Appointments are not necessary for episodic health needs. Usually open 7 days a week, RHCs offer extended hours on weekdays. ${ }^{2}$

The clinics are staffed by licensed, qualified advance practice providers, such as nurse practitioners and physician assistants, who are supervised by family physicians where required by state law. These clinicians have advanced education to diagnose, treat, and prescribe for nonemergent ailments such as colds and flu, rashes and skin irritation, and muscle strains or sprains. ${ }^{3}$ They are supported

Mr. Bronstein is Chief Operating Officer, Convenient Care Association, Philadelphia, Pennsylvania.

Dr. Rayburn is Emeritus Distinguished Professor and Chair, Department of Obstetrics and Gynecology, University of New Mexico School of Medicine, Albuquerque; and Adjunct Professor, Medical University of South Carolina, Charleston.

The authors report no financial relationships relevant to this article.

doi: $10.12788 /$ obgm.0086 by an electronic health record that contains established evidence-based protocols. ${ }^{2,4}$

\section{Evolution of retail health clinics} The first RHC, operated by QuickMedx, opened its doors in 2000 in MinneapolisSt. Paul. ${ }^{1,5}$ Only patients with a very limited number of illnesses were seen, and payment was cash. In 2005, this clinic was acquired by a major pharmacy retailer, which led to several acquisitions by other retailers and health care systems. In addition to accepting cash for a visit, the clinics formed contracts with health insurance companies. The average cost of a visit to an RHC in 2016 was estimated to be $\$ 70$, considerably less than the cost at urgent care clinics (\$124) and emergency rooms $(\$ 356){ }^{6}$

Today, more than 20 companies provide health care services at RHCs (TABLE 1, page 32). CVS (MinuteClinic) has the most retail clinics, followed by Walgreens, Kroger, Rite Aid, and Zoom+Care., ${ }^{1,2}$ There are now about 3,300 clinics in the United States, Canada, and Mexico (nearly all are located in the United States). ${ }^{2}$ Currently, RHCs are present in 44 states and the District of Columbia. Alabama, Alaska, Idaho, North Dakota, Vermont, and Wyoming are the only states without an RHC, while large-population states (California, Florida, Ohio, Pennsylvania, and Texas) have experienced an explosion in clinic openings. ${ }^{2}$
IN THIS ARTICLE

$\mathrm{RHC}$ services

page 32

Differences in episodic care clinics page 33

ObGyn-RHC interaction page 34 
TABLE 1 Approximate numbers of retail health clinics for each operator in the United States, 2019

\begin{tabular}{l|c}
\hline Retail health clinic & Number \\
\hline CVS, MinuteClinic & 1,160 \\
\hline Walgreens & 400 \\
\hline Kroger, The Little Clinic & 283 \\
\hline Rite Aid, RediClinic & 75 \\
\hline Hy-Vee & 69 \\
\hline Zoom+Care & 43 \\
\hline BellinHealth FastCare & 36 \\
\hline Lindora Clinic & 34 \\
\hline Piedmont Healthcare & 28 \\
\hline Walmart & 25 \\
\hline Sutter Health & 12 \\
\hline Baptist Health Express Care & 12 \\
\hline Aurora Quickcare & 8 \\
\hline Source: Convenient Care Association. 2020 Retail Health \\
\hline Capacity Report (white paper).
\end{tabular}

RHCs are found in high foot traffic locations, such as large retailers and grocery stores, and in prioritizing services such as drug stores. By analyzing 2019 clinic openings and population centers, the Convenient Care Association determined that more than half of the US population now lives within a 10-minute drive of an RHC. ${ }^{2}$ New locations are established on a regular basis, resulting in some flux in the total number of clinics.

\section{Services that RHCs provide}

As RHC locations expand, so do their services. Most RHCs pursue 1 of 2 models: health hubs or virtual care. Health hubs offer an expansion of services, which has resulted in retail health looking and operating more like the primary care providers in their communities. ${ }^{1,2}$ While most chains intend for patient visits to be brief, the growing health hub model intends to expand the period for patient visits. The major companies, CVS, Kroger, and Walmart, are offering an increase in their services and granting their providers a greater capacity to screen and treat patients for a wider range of conditions. By contrast, other clinic operators, such as Rite Aid RediClinics, are pursuing a more episodic and convenient care model with a greater adoption and expansion of telehealth and telemedicine.

Services at RHCs involve primarily acute care as well as some basic chronic disease management. About $90 \%$ of visits are for the following conditions: influenza, immunizations, upper respiratory infections, sinusitis, bronchitis, sore throat, inner ear infection, conjunctivitis, urinary tract infections, and blood tests. ${ }^{1-3}$ Other services available at most RHC locations involve screening and monitoring, wellness and physicals, travel health, treatment of minor injuries, and vaccinations and injections.

Women constitute half of all customers, and all RHCs offer women's health services. ${ }^{7}$ Along with addressing acute care needs, women's health services include contraception care and options, human papillomavirus (HPV) screening, pregnancy testing and initial prenatal evaluation, and evaluation for and treatment of urinary tract, bladder, and yeast infections. ${ }^{2,6}$

All RHCs provide counseling on sexual health concerns. Nearly all retail clinics in the United States provide screening and treatment for patients and their partners with sexually transmitted infections. RHC providers are required to follow up with patients regarding any blood work or culture results. When positive test results are confirmed for serious infections, such as hepatitis B and C, syphilis, and HIV, patients customarily are referred for treatment. ${ }^{2}$

\section{The RHC patient base}

RHCs serve an expanding base of patients who cite convenience as their primary motivation for utilizing these clinics. This consumer-driven market now encompasses, by some measures, nearly 50 million visits annually. ${ }^{2}$ These numbers have risen every year alongside a consistent increase in the number and spread of clinics across the country. During the COVID-19 pandemic, visits declined, consistent with other health care touchpoints, due to concerns about spreading the coronavirus. 
TABLE 2 Differences between episodic care clinics

\begin{tabular}{|c|c|c|c|}
\hline & Retail health clinics & Urgent care clinics & Emergency departments \\
\hline Business hours & Extended, weekends & Extended, weekends & $24 / 7$ \\
\hline Types of conditions & Acute, chronic & Acute & Acute, life-threatening \\
\hline Provider & NPs, PAs & NPs, PAs, physicians & Physicians \\
\hline Primary facility & Stand-alone, retail & Stand-alone & Hospital \\
\hline Medical costs & $\$$ & $\$ \$$ & $\$ \$ \$$ \\
\hline
\end{tabular}

The RHC industry has continued to adapt to a changing health care climate by embracing new telehealth solutions, enabling remote care, and expanding services by consumer demand. ${ }^{1,7}$ While convenience is a primary motivation for visiting an RHC, about two-thirds of RHC patients do not have a primary care provider. ${ }^{1}$ To support a broader continuum of care, RHCs regularly refer patients who do not have a primary care provider to other health care touchpoints when necessary.

Young and middle-aged adults (18-44 years) comprise the largest group of RHC patients. When patients were asked why they chose an RHC over 'traditional doctors' clinics," many cited difficulties in accessing care, the appeal of lower costs, and proximity. The proportion of female RHC users was 50.9\% and $56.8 \%$ for RHC nonusers. ${ }^{1-3}$

\section{How RHCs compare with other episodic care clinics}

The consumer has more choices to seek episodic care other than at physicians' clinics or emergency rooms. An RHC, urgent care clinic, or freestanding emergency clinic increase access points for consumers. Along with the expanding number of RHCs, there are nearly 9,000 US urgent care centers, according to the Urgent Care Association, and more than 550 freestanding emergency rooms. ${ }^{8}$

The main differences between these episodic care clinics are shown in TABLE 2. Hours of operation, types of conditions, available providers, location of the facility, and estimated costs are compared. All provide expanded business hours. Retail clinics address some chronic disease management along with acute care, engage only advanced practitioners, use retail stores, and are less costly to consumers. ${ }^{3,49}$

An RHC, urgent care clinic, or emergency department increases access points for consumers. Many emergency department visits can be handled in ambulatory settings such as RHCs and urgent care clinics. ${ }^{9,10}$ This can be helpful, especially in rural areas with a shortage of physicians. Most people want a relationship with a physician who will manage their care rather than seeing a different provider at every visit. While ObGyns deliver comprehensive care to women, however, in some underserved areas non-ObGyn clinics can fill the void. For example, RHCs can sometimes provide needed immunizations and health care information required in underserved areas. ${ }^{11}$

Many physicians are frustrated when they see patients who do not have a complete copy of their medical record and must piecemeal how to treat a patient. RHCs have adopted electronic medical records, and they regularly encourage patients to contact their physician (or find one, which can be difficult). Another limitation can be a referral from an RHC to a subspecialist rather than a primary care physician who could equally handle the condition.

\section{What ObGyns can do}

Consumers have become accustomed to obtaining services where and when they want them, and they expect the same from their health care providers. While ObGyn

practices are less affected by RHCs than

\begin{tabular}{l}
\hline FAST \\
TRACK \\
\hline Strong \\
relationships \\
between ObGyns \\
and their patients \\
and exceptional \\
overall service can \\
form the bonds, \\
and RHCs may \\
provide back-up \\
support
\end{tabular}

\section{FAST}

TRACK 
family physicians or general internists, health care delivery in traditional clinics must be user friendly - that is, better, cheaper, and faster-for the patient-consumer to be more satisfied. Looking ahead, a nearby women's health care group needs to have someone on call 24 hours a day, 7 days a week. That way, you can tell your patients that they can call you first if they need help. In the case of an ObGyn recommending that a patient go to an RHC or urgent care center, you will be aware of the visit and can follow up with your patient afterward.

Traditional clinics need to create ways for patients with acute illnesses to be seen that same day. Offering extended hours or technology options, such as online support, can help. Text message reminders, same-day access for appointments, and price transparency are necessary. It is important to encourage your women's health patients to become more responsible for their own health and care, while taking into consideration their social determinants of health. While ObGyns should discuss with their patients when to visit an RHC (especially when their clinic is closed), emphasize that your own clinic is the patient's medical home and encourage the importance of communicating what occurred during the RHC visit.

Working as a team by communicating well can create a community of health. It would be appropriate for you to represent your group by meeting practitioners at the nearby RHC. Being accessible and helpful would create a friendly and open professional relationship. Conversely, providers at retail clinics need to continually appreciate that women's health clinics offer more comprehensive care. Select referrals from RHCs would help the most important person, the patient herself.
References

1. Bachrach D, Frohlich J, Garcimonde A, et al. Building a culture of health: the value proposition of retail clinics. Robert Wood Johnson Foundation and Manatt Health. April 2015. https://www.rwjf.org/en/library/research/2015/04/ the-value-proposition-of-retail-clinics.html. Accessed March 26, 2021.

2. Bronstein N. Convenient Care Association-National Trade Association of Companies and Healthcare Systems for the Convenient Care Industry, January 1, 2020. https://www. ccaclinics.org/about-us/about-cca. Accessed January 10, 2021.

3. Mehrotra A, Liu H, Adams JL, et al. Comparing costs and quality of care at retail clinics with that of other medical settings for 3 common illnesses. Ann Intern Med. 2009;151:321-328.

4. Woodburn JD, Smith KL, Nelson GD. Quality of care in the retail health care setting using national clinical guidelines for acute pharyngitis. Am J Med Qual. 2007;22:457-462.

5. Zamosky L. What retail clinic growth can teach physicians about patient demand. Threat or opportunity: retail clinic popularity is about convenience. Med Econ. 2014;91: $22-24$
6. SolvHealth website. Urgent care center vs emergency room https://www.solvhealth.com/faq/urgent-care-center-vsemergency-room. Accessed January, 13, 2021.

7. Kvedar J, Coye MJ, Everett W. Connected health: a review of technologies and strategies to improve patient care with telemedicine and telehealth. Health Aff. 2014;33:194-199.

8. Urgent Care Association website. Industry news: urgent care industry grows to more than 9,000 centers nationwide. February 24, 2020. https://www.ucaoa.org/About-UCA/ Industry-News/ArtMID/10309/ArticleID/1468/INDUSTRYNEWS-Urgent-Care-Industry-Grows-to-More-than-9000Centers-Nationwide. Accessed March 26, 2021.

9. Sussman A, Dunham L, Snower K, et al. Retail clinic utilization associated with lower total cost of care. Am J Manag Care. 2013;19:e148-57.

10. Weinik RM, Burns RM, Mehrotra A. Many emergency department visits could be managed at urgent care centers and retail clinics. Health Aff. 2010;29:1630-1636.

11. Goad JA, Taitel MS, Fensterheim LE, et al. Vaccinations administered during off-clinic hours at a national community pharmacy: implications for increasing patient access and convenience. Ann Fam Med. 2013;11:429-436. 